\title{
Einführung von RFID in der WLB
}

Radio Frequency Identification, RFID, wird mittlerweile in vielen, zunehmend auch in wissenschaftlichen Bibliotheken eingesetzt, vor allem um die Vorteile von Selbstverbuchung und Rückgabeautomation zu nutzen.

So nun auch in der WLB. Die Entscheidung für RFID wurde bereits im November 2010 getroffen - im Hinblick auf den im Erweiterungsbau vorgesehenen Freihandbereich, der 500.000 Bände fassen soll. Als Anfangsbestand, d. h. pünktlich zum Eröffnungstermin im August 2015, wollen wir dort insgesamt 200.000 Bände frei zugänglich anbieten, beginnend mit dem Jahresring 55/ff (Monografien in Oktav- und Quartformat, keine Sonderkontingente).

Da wir spätestens zu diesem Termin auf Selbstverbuchung und einen Rückgabeautomaten mit Sortieranlage setzen, sind darüber hinaus aber auch alle Medien auszustatten, die von unseren Benutzer(inne)n aus den Magazinen bestellt und im Selbstabholerbereich bereitgelegt werden. Nachdem Geschäftsgangs- und konkrete Ausstattungsfragen geklärt waren, die Hard- und Software geliefert wurde, konnte das Tagging beginnen. Der Startschuss erfolgte am 2.2.2011.

Seither wird einerseits der komplette Neuzugang (Jahresring 61/ff) mit den entsprechenden Tags ausgestattet. Daran beteiligt ist die Schlussstelle für alle die Medien, die noch weitere Geschäftsgangsstationen (z. B. Sacherschließung, Landesbibliographie, Sonderstandorte) durchlaufen müssen, die Medienbearbeitung (MB 3) für die nonbook-Materialien, die MB 4 für die Zeitschriften. Den größten Anteil bewältigt die Leihstelle, da sie alle Medien bearbeitet, die von der Schlussstelle aus direkt ins Magazin gehen.

Und andererseits werden die Jahresringe 55/ff und 55a/ff, die 2015 vom Tiefmagazin in den Freihandbereich zu transferieren sind, ausgestattet. Systematisch arbeitet ein Team im Magazin am Regal entlang, parallel dazu stattet die Leihstelle den Rücklauf aus.
Nach unserer bisherigen Erfahrung mit der Ausstattung durch Kolleg(inn)en des Magazins, die verstärkt durch Kolleg(inn)en der Leihstelle immer dann fleißig Tags kleben und Daten konvertieren, wenn es die Zeit zulässt, mussten wir feststellen, dass die großen Bestände "so nebenbei" doch nicht zu erfassen sind. Aus diesem Grund haben wir Ende Juli zwei zusätzliche Aushilfskräfte eingestellt, die ausschließlich zur RFID-Ausstattung eingesetzt werden.
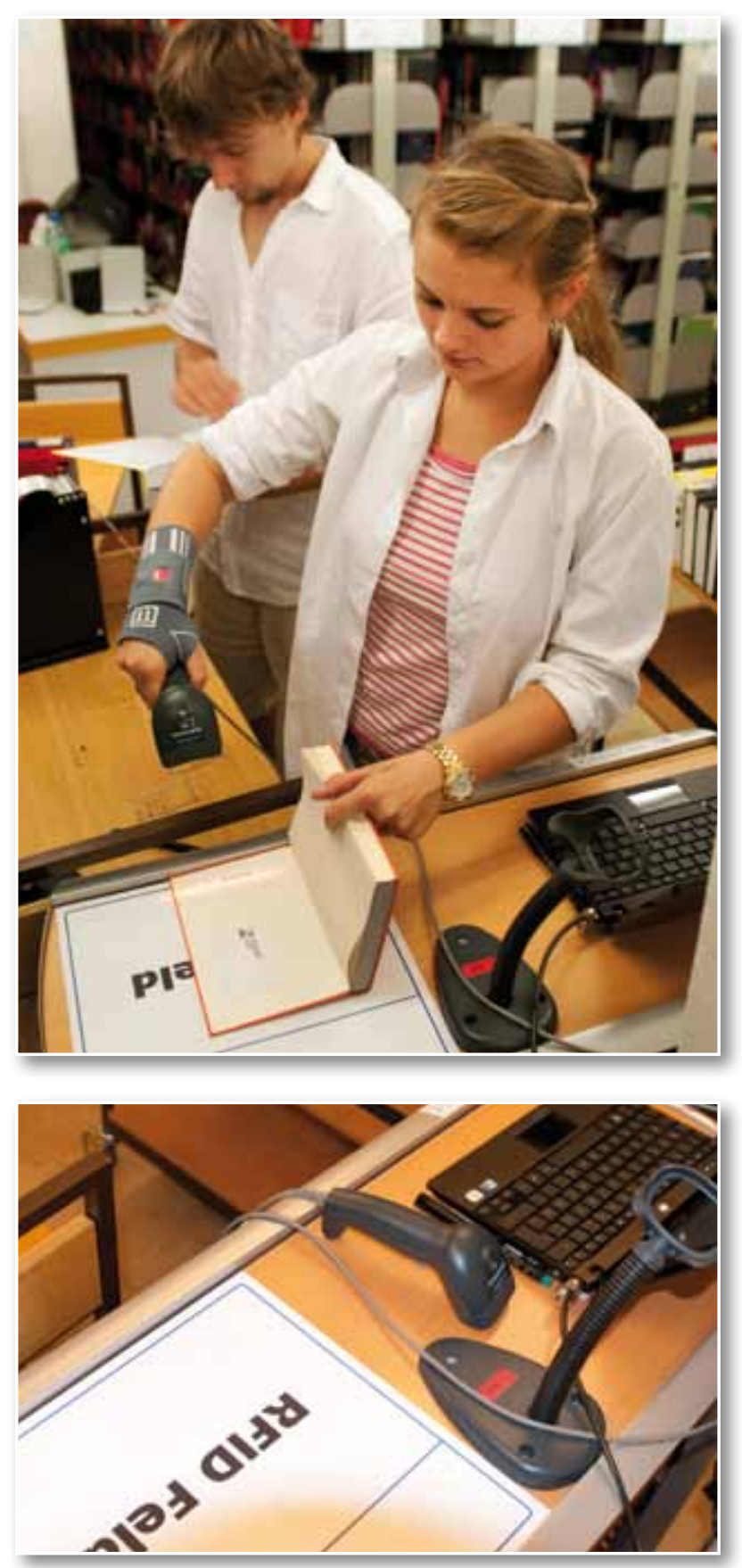


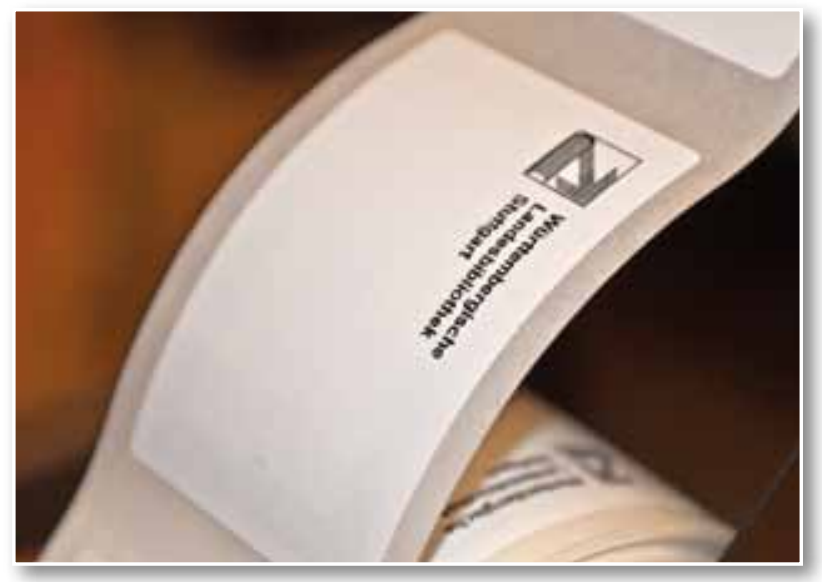

Wir statten nun alle Medien aus, aber wird die RFID-Technik im Erweiterungsbau auch so funktionieren, wie wir uns das vorstellen? Das ist die große Frage, auf deren Beantwortung wir nicht erst bei der feierlichen Eröffnung des Erweiterungsbaus im Jahr 2015 warten wollen. Deshalb werden wir, wenn das nächste Großprojekt - die Migration auf aDis/BMS - voraussichtlich im Sommer 2013 abgeschlossen sein wird, den Selbstabholerbereich zur Trainingsplattform umwidmen und dort mit der Selbstverbuchung beginnen. 2013 wird in vielerlei Hinsicht ein aufregendes Jahr werden.

Martina Lüll

\section{LEO-BW - Landeskunde entdecken online Das vernetzte landeskundliche Informationssystem für}

\section{Baden-Württemberg}

\section{BLO, LAGIS, Sachsen.digital, KuLaDigNW, OPAL} Niedersachsen, Internet-Portal "Westfälische Geschichte", Regionalgeschichte.net, HamburgWissen digital ${ }^{1}$.... Portale im Internet boomen! Das sind nur einige landeskundliche und landesgeschichtliche Informationsportale in Deutschland. Diese Portale wurden und werden vielerorts geplant, realisiert, weiterentwickelt oder feiern wie die Bayerische Landesbibliothek Online schon ihr 10jähriges Jubiläum. Zudem haben sich zahlreiche Institutionen mit ihren vielfältigen Internetangeboten in der Arbeitsgemeinschaft landesgeschichtlicher und landeskundlicher Internet-Portale in Deutschland ${ }^{2}$ zusammengeschlossen. Nicht mehr nur das Angebot einer zentralen Suchoberfläche zu einem Themengebiet steht hier im Vordergrund, sondern die Vernetzung der einzelnen Internetangebote untereinander.

Nun hat auch Baden-Württemberg ein Wissensportal: LEO-BW ist da! Ein landeskundliches Informationssystem der besonderen Art. Als Geschenk zum Landesjubiläum 2012 ist es seit dem 25. April mit 1,36 Mio. Objekten freigeschaltet. Doch der Weg dahin war lang.

\section{Projektgeschichte und Eckdaten}

Die Ideen für ein landeskundliches Informationssystem gehen sowohl bei den beiden Landesbibliotheken in Baden-Württemberg, der Badischen Landesbibliothek in Karlsruhe und der Württembergischen Landesbibliothek in Stuttgart, als auch beim Landesarchiv Baden-Württemberg bis ins Jahr 2002 zurück. Denn baden-württembergische Institutionen stellen zahlreiche interessante und vielfältige Informationen, Materialien, Quellen und Literatur über Baden-Württemberg im Internet bereit. Doch mussten Nutzer die benötigten Informationen einzeln über diese Institutionen mit unterschiedlichsten Suchoberflächen recherchieren. Auf der Basis eines Vorprojektes, in dem ein technisches Grobkonzept entwickelt und eine Kostenkalkulation durchgeführt wurde, erfolgte durch das Landesarchiv Baden-Württemberg ein Förderan-

(1) Bayerische Landesbibliothek Online (BLO): http://www.bayerische-landesbibliothek-online.de Landesgeschichtliches Informationssystem Hessen: http://lagis.online.uni-marburg.de Sachsen.digital: http://www.sachsendigital.de KuLaDigNW: http://www.kuladignw.de/KuLaDigNW/ OPAL Niedersachsen: http://opal-niedersachsen.de Internet-Portal "Westfälische Geschichte": http://www.westfaelische-geschichte.Iwl.org Regionalgeschichte.net: http://www.regionalgeschichte.net HamburgWissen digital [in Vorbereitung]: http://www.hamburgwissen-digital.de

(2) Arbeitsgemeinschaft landesgeschichtlicher und landeskundlicher Internet-Portale in Deutschland: 